\title{
Electron capture to the continuum in collisions of bare projectiles with $\mathrm{Ne}$ targets
}

\author{
W Oswald, R Schramm, D H Jakubassa-Amundsen and H-D Betz \\ Sektion Physik, Universitāt München, 85748 Garching, Federal Republic of Germany
}

Received 22 June 1993, in final form I4 October 1993

\begin{abstract}
We have investigated the cusp resulting from electron capture to the continuum of $1.25-5 \mathrm{MeV} \mathrm{amu}^{-1}$ fully stripped hydrogen and oxygen as a function of the collision energy and the detector angular resolution $\theta_{0}$. It is revealed that the characteristic cusp shape parameters depend strongly on the experimental resolution. Our experimental data are compared with the second-order Born theory and the impulse approximation. Both theories confirm the $\theta_{0}$ dependence of the shape parameters and give a reasonable description of the cusp asymmetry. However, theory tends to overestimate the absolute cross sections, in particular in the case of oxygen.
\end{abstract}

\section{Introduction}

When fully stripped ions collide with target atoms, electrons can be captured into the continuum (ECC) of the projectile. These electrons travel with approximately the same velocity as the projectile and hence appear as a cusp-shaped peak in the spectra recorded at zero emission angle with respect to the beam direction. Since the discovery of the ECC electrons (Rudd et al 1966), much work has been devoted to the study of the peak shape (for a review see Groeneveld et al 1984). Salin (1969) and Macek (1970) were the first to recognize that the cusp phenomenon is related to the properties of a nearthreshold projectile eigenstate. A simple first-order ECC approach was sufficient to show that the doubly differential cross section with respect to the electron momentum $k_{\mathrm{f}}$ and emission angle $\vartheta_{\mathrm{f}}$ actually is divergent in the cusp (at $k_{\mathrm{f}}=v$ and $\vartheta_{\mathrm{f}}=0$, where $v$ is the projectile velocity). From this consideration it became obvious that the cusp is strongly dependent on the acceptance angle $\theta_{0}$ of the electron detector.

Since theory has revealed simple scaling properties with $\theta_{0}$ (Dettmann et al 1974, Day 1980) as far as the cusp width and peak intensity are concerned, experiments have nearly exclusively been carried out at a fixed $\theta_{0}$. On the other hand, Mecbach et al (1981) suggested disentangling the $\theta_{0}$ dependence of the cusp shape by defining shape parameters $B_{n l}$ in the projectile rest frame which ought to be independent of $\theta_{0}$. These parameters are readily extracted from experiment by transforming the parametrized cross section into the target frame, folding it with the detector resolution and fitting it to the experimental spectra.

Systematic ECC experiments (Breinig et al 1982, Knudsen et al 1986, Gulyás et al 1986) have revealed a strong cusp asymmetry which is at variance with the first-order Born theory. This cusp asymmetry could only be explained within a second- or higherorder approach. The necessity of a higher-order approach originates from ECC being a 
rearrangement process where (from capture-to-bound-state studies) the failure of a first Born approach is well established. It had not been realized, however, that the degree of asymmetry depends on the chosen value of $\theta_{0}$.

Several higher-order approaches have been used for the description of the cusp electrons, symmetric theories like the second-order Born approximation (B2; Dettmann et al 1974, Shakeshaft and Spruch 1978, Miraglia and Ponce 1980, Moiseiwitsch 1991) and the continuum distorted wave approach (Garibotti and Miraglia 1980, Burgdörfer 1986, Crothers and McCann 1987) and, on the other hand, the impulse approximation (IA) in its post and prior form, which is suited for asymmetric collision systems (Jakubassa-Amundsen 1983, 1988).

All these theories reveal a discontinuity in the projectile-rest-frame cross section across the cusp. When transformed in to the target frame, this leads to a strong enhancement of the intensity on the low-energy side of the cusp $\left(k_{\mathrm{f}}<v\right)$. Hence, in contrast to previous assumptions, it is no longer guaranteed that shape parameters extracted from experiment are indeed independent of the detector resolution.

The present experiments were designed to examine the shape parameters when the experimental resolution is both improved and varied over a certain range. A brief report of our investigations has been given earlier (Oswald et al 1989). In previous experiments, $\theta_{0}$ was in general around $2-3^{\circ}$ and mostly $\mathrm{H}$ and $\mathrm{He}$ targets have been used apart from a few early experiments on ECC from $\mathrm{Ne}$ and $\mathrm{Ar}$ (Cranage and Lucas 1976, Menendez et al 1977, Rødbro and Andersen 1979). We have carried out measurements with $\theta_{0}$ ranging from $0.1^{\circ}$ to $1.2^{\circ}$ and we have used heavy targets like $\mathrm{CH}_{4}$ and $\mathrm{Ne}$ where L-shell electrons contribute considerably to ECC at the lower collision velocities. Also we have used two different projectiles, $\mathrm{H}^{+}$and $\mathrm{0}^{8+}$, in order to study the projectile dependence of the shape parameters. In the case of a helium target it had been suggested that the $B_{n l}$ obey scaling relations with the projectile charge (Knudsen et al 1986).

For the theoretical explanation the second-order Born theory and the impulse approximation is applied. Previous $\mathrm{B} 2$ investigations had been restricted to $\mathrm{H}$ and $\mathrm{He}$ targets, and a series of peaking approximations had been introduced in order to cast the formalism into a numerically tractable form. These approximations provide the correct high-energy limit but will fail when the conditions $v \gg Z_{\mathrm{P}}$ and $v \gg Z_{\mathrm{T}}$ (where $Z_{\mathrm{P}}$ and $Z_{\mathrm{T}}$ are the nuclear charges of the projectile and target, respectively) are violated. The peaking approximations affect both magnitude and relative phase of the first and second Born term in the capture amplitude, resulting in a considerable sensitivity of the shape parameters on these approximations. In this work the second-order Born theory is applied without peaking approximations, including in the interaction potential the screening by the passive target electrons. Our 82 results are an improvement on earlier calculations (Oswald 1989, Oswald et al 1989) where screening had been neglected. For comparison, calculations have also been performed with the fully peaked prior impulse approximation (for $\mathrm{H}^{+}$on $\mathrm{Ne}$ ) and with the transverse peaked post impulse approximation (for $\mathrm{O}^{8+}$ on $\mathrm{Ne}$ ).

The paper is organized as follows. In section 2 , the experimental details are described. Section 3 contains the basic formalism of the second-order Born theory and in section 4 the impulse approximation is briefly outlined. Section 5 gives a comparison of the experimental spectra with theory, and in section 6 the cusp shape is discussed for different detector resolutions. The work is concluded with some final remarks (section 7). Atomic units $(\hbar=m=e=1)$ are used unless otherwise indicated. 


\section{Experiment}

The Munich and Heidelberg tandem accelerator facilities have been used to prepare beams of $25-80 \mathrm{MeV}$ bare oxygen ions and 1.25-4.2 MeV protons, respectively. Beam normalization was achieved using a Faraday cup, the output of which was fed into a current digitizer consisting of a fast current-to-voltage converter and a voltage-tofrequency converter.

The gas target was a differentially pumped cell of $8 \mathrm{~mm}$ length operated with neon gas. The vacuum in the chamber did not exceed $10^{-5}$ Torr when the target was in operation. The signal was found to vary linearly with target pressure indicating that single-collision conditions were fulfilled.

The electrons were analysed using a magnetic spectrometer. Our experimental setup is shown schematically in figure 1. In the Munich experiments (for oxygen projectiles)

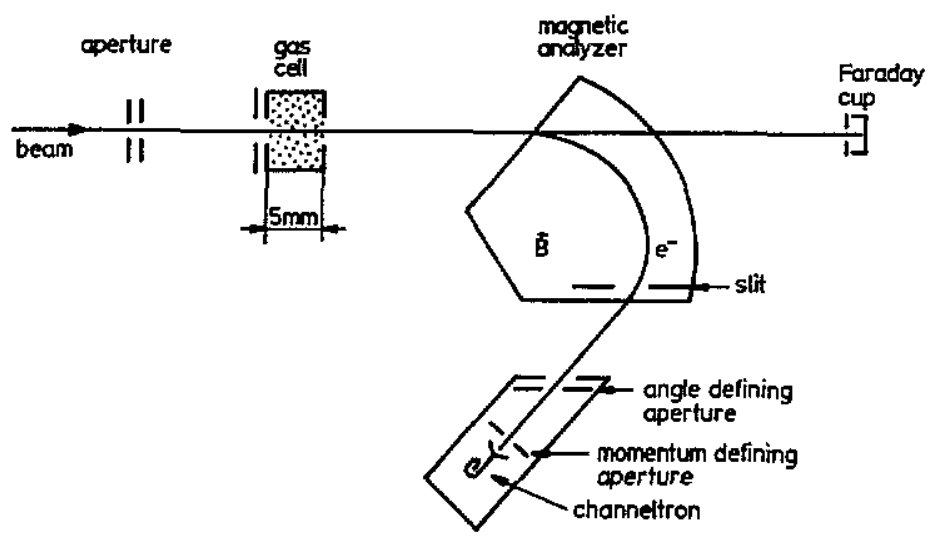

Figure 1. Schematic view of the experimental set-up.

background effects were minimized by applying $-300 \mathrm{~V}$ bias to the front electrode of the channeltron detector and by careful design and positioning of all apertures. Additionally, a transverse field of some $100 \mathrm{~V} \mathrm{~cm}^{-1}$ was applied in front of the gas cell in order to deffect electrons produced at the beam collimating apertures. The magnetic field of the earth and stray fields were reduced to less than $0.05 \mathrm{G}$ using mumetal shielding.

As it was an important aim of our work to study the shape of the ECC peak and its dependence on the experimental angular resolution, special care was taken in designing our magnetic spectrometer. Based on the work of Enge (1967) on focusing of charged particles, the properties of our analyser could be obtained from geometry using ray-tracing techniques. In our trajectory calculations we have also accounted for aberration and included the effect of the finite target size (Oswald 1989). The results of the calculations are summarized as follows.

The resolution properties may be expressed by a single momentum resolution $\Delta p /$ $p$ and a single angular resolution, $\theta_{0}$, which are independent of each other if, and only if, two conditions are fulfilled. Firstly, the beam divergence has to be much smaller than the angular resolution $\theta_{0}$, and secondly the following inequality must hold 
where $d$ is the beam diameter, $l$ is the target length and $R$ is the radius of the path of the electrons through the magnet. With a beam divergence of less than $0.05^{\circ}, d=$ $0.8 \mathrm{~mm}, l=8 \mathrm{~mm}$ and $R=55 \mathrm{~mm}$, these requirements were excellently met in our experiments, freeing us from the necessity of folding procedures with somehow constructed transmission functions.

Using appropriately sized circular apertures, the momentum resolution of the apparatus was determined to be $\Delta p / p=2.5 \times 10^{-3}$ and the angular resolution was variable from $0.1^{\circ}$ to $1.2^{\circ}$. These values were additionally verified by resolving a series of known KLL Auger lines from $\mathrm{O}^{5+}$ projectiles (Stolterfoht 1978).

\section{Second-order Born theory}

For the theoretical description of the capture of a target electron by a bare projectile, the single-particle model is used and the passive electrons are accounted for by a screened target potential. For energetic collisions the nucleus-nucleus interaction may be neglected such that the wavefunctions for the internuclear motion can be represented by plane waves. This is equivalent to a semiclassical description with a straight-line internuclear trajectory. For rearrangement processes like ECC, the decomposition of the Hamiltonian into an unperturbed part $H_{0}$ and a perturbation $V$ is different in the initial and final channels. The unperturbed part consists of the kinetic energies $T_{\mathrm{N}}$ and $T_{\mathrm{c}}$ for the internuclear and the electronic motion, respectively. For an initially bound target electron in the state $\phi_{\mathrm{i}}^{\mathrm{T}}, H_{\mathrm{Oi}}$ includes the interaction $V_{\mathrm{T}}$ of the active electron with the target core while in the final channel, where the electron is in a projectile eigenstate $\phi_{\mathrm{f}}^{\mathrm{p}}$, the Coulomb field $V_{\mathrm{P}}$ between electron and projectile is incorporated into $H_{0 \mathrm{of}}$. However, to allow for an analytical evaluation of major parts of the transition amplitude a hydrogenic eigenstate is chosen for $\phi_{\mathrm{i}}^{\mathrm{l}}$. Accordingly, $V_{\mathrm{T}}$ is split into two parts (Newton 1966): one part, the pure Coulomb field $V_{\mathrm{T}}^{\mathrm{C}}$, is included in $H_{0 \mathrm{i}}$ while the second part, $V_{\mathrm{T}}^{\mathrm{S}}$, which accounts for the screening by the passive electrons is treated as perturbation together with the projectile field $V_{\mathrm{P}}$. In the final state both parts of $V_{\mathrm{T}}$ are treated perturbatively. Explicitly

$$
\begin{array}{lll}
H=H_{01}+V_{\mathrm{i}} & H_{0 \mathrm{i}}=T_{\mathrm{N}}^{\prime}+T_{\mathrm{c}}^{\prime}+V_{\mathrm{T}}^{\mathrm{C}}\left(r^{\prime}\right) & V_{\mathrm{i}}=V_{\mathrm{P}}(\boldsymbol{r})+V_{\mathrm{T}}^{\mathrm{S}}\left(\boldsymbol{r}^{\prime}\right) \\
H=H_{0 \mathrm{f}}+V_{\mathrm{r}} & H_{0 \mathrm{f}}=T_{\mathrm{N}}+T_{\mathrm{e}}+V_{\mathrm{P}}(\boldsymbol{r}) & V_{\mathrm{f}}=V_{\mathrm{T}}\left(r^{\prime}\right) \equiv V_{\mathrm{T}}^{\mathrm{C}}\left(\boldsymbol{r}^{\prime}\right)+V_{\mathrm{T}}^{\mathrm{s}}\left(\boldsymbol{r}^{\prime}\right)
\end{array}
$$

with the definitions

$$
V_{\mathrm{P}}(r)=-\frac{Z_{\mathrm{P}}}{r} \quad V_{\mathrm{T}}^{\mathrm{c}}\left(r^{\prime}\right)=-\frac{Z_{\mathrm{T}}}{r^{\prime}} \quad V_{\mathrm{T}}^{\mathrm{s}}\left(r^{\prime}\right)=\frac{Z_{\mathrm{T}}-1}{r^{\prime}}\left(1-\mathrm{e}^{-\lambda r^{\prime}}\right)
$$

where $Z_{\mathrm{P}}$ and $Z_{\mathrm{T}}$ are the charges of the projectile and the target nucleus, respectively, and the functional form of $V_{\mathrm{T}}^{\mathrm{S}}$ is chosen such that the electron-core interaction $V_{\mathrm{T}}$ has the correct $-1 / r^{\prime}$ behaviour at large distances. The screening constant $\lambda$ is determined from the requirement that the expectation value of $T_{\mathrm{c}}^{t}+V_{\mathrm{T}}$ in the initial state $\phi_{\mathrm{i}}^{\mathrm{T}}$ is equal to the experimental energy $E_{i}^{\mathrm{T}}$ of the initial state,

$$
\lambda=2 Z_{\mathrm{T}}\left[\left(\frac{Z_{\mathrm{T}}\left(Z_{\mathrm{T}}-1\right)}{Z_{\mathrm{T}}^{2} / 2-Z_{\mathrm{T}}-E_{\mathrm{i}}^{\mathrm{T}}}\right)^{1 / 2}-1\right] .
$$




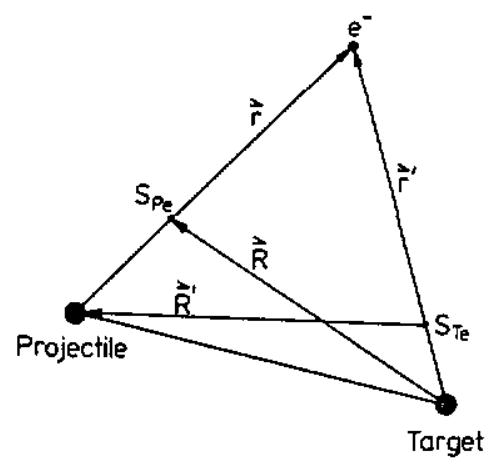

Figure 2. Coordinates for the collision system consisting of the projectile, the target core and the active electron. The centre of mass of the projectile-electron system is denoted by $S_{\mathrm{P}_{\mathrm{e}}}$, that of the electron-target core system by $S_{\mathrm{Te}}$.

Note, that for a hydrogen target $\left(Z_{\mathrm{T}}=1\right)$ the screening potential $V_{\mathrm{T}}^{\mathrm{S}}$ is absent. In order to include the recoil effects correctly, different coordinate systems have to be used in the initial and in the final channel (distinguished by a prime, see figure 2). This is equivalent to the inclusion of translational factors in the semiclassical approach.

In the second-order Born theory the transition matrix element is given by

$$
\begin{aligned}
& T_{\mathrm{f}_{\mathrm{i}}}=T_{\mathrm{l}}+T_{2} \\
& T_{\mathrm{l}}=\left\langle\boldsymbol{K}_{\mathrm{f}} \phi_{\mathrm{f}}^{\mathrm{P}}(\boldsymbol{r})\left|V_{\mathrm{f}}\right| \boldsymbol{K}_{\mathrm{i}}^{\prime} \phi_{\mathrm{s}}^{\mathrm{T}}\left(\boldsymbol{r}^{\prime}\right)\right\rangle \\
& T_{2}=\int \mathrm{d} \boldsymbol{K}^{\prime} \mathrm{d} \boldsymbol{k}^{\prime}\left\langle\boldsymbol{K}_{\mathrm{f}} \phi_{\mathrm{f}}^{\mathrm{P}}(\boldsymbol{r})\left|V_{\mathrm{f}}\right| \boldsymbol{K}^{\prime} \boldsymbol{k}^{\prime}\right\rangle \frac{1}{E_{\mathrm{i}}^{\text {tot }}-E_{0}+\mathrm{i} \varepsilon}\left\langle\boldsymbol{K}^{\prime} \boldsymbol{k}^{\prime}\left|V_{\mathrm{i}}\right| \boldsymbol{K}_{\mathrm{i}}^{\prime} \phi_{\mathrm{i}}^{\mathrm{T}}\left(\boldsymbol{r}^{\prime}\right)\right\rangle
\end{aligned}
$$

where $\left|\boldsymbol{K}_{\mathrm{i}}^{\prime}\right\rangle=(2 \pi)^{-3 / 2} \exp \left(\mathrm{i} \boldsymbol{K}_{\mathrm{i}}^{\prime} \boldsymbol{R}^{\prime}\right)$ and $\left|\boldsymbol{K}_{\mathrm{f}}\right\rangle=(2 \pi)^{-3 / 2} \exp \left(\mathrm{i} \boldsymbol{K}_{\mathrm{f}} \boldsymbol{R}\right)$ are the initial and final states of the internuclear motion with the corresponding momenta $K_{1}$ and $K_{\mathrm{f}}$, respectively. A complete set of nuclear $\left(\left|\boldsymbol{K}^{\prime}\right\rangle\right)$ and electronic $\left(\left|\boldsymbol{k}^{\prime}\right\rangle\right)$ plane waves has been introduced into the second Born term $T_{2}$ in order to allow for a straightforward evaluation of the free propagator $\left(E_{\mathrm{i}}^{\text {tot }}-H_{0}+\mathrm{i} \varepsilon\right)^{-1}\left|\boldsymbol{K}^{\prime} \boldsymbol{k}^{\prime}\right\rangle=\left(E_{\mathrm{i}}^{\text {tot }}-E_{0}+\mathrm{i} \varepsilon\right)^{-1}\left|\boldsymbol{K}^{\prime} \boldsymbol{k}^{\prime}\right\rangle$ with $E_{0}=$ $K^{\prime 2} / 2 v^{\prime}+k^{\prime 2} / 2 \beta$ and the total energy $E_{\mathrm{i}}^{\text {tot }}=E_{\mathrm{f}}^{\text {tot }}=K_{\mathrm{i}}^{\prime 2} / 2 v^{\prime}+E_{\mathrm{i}}^{\mathrm{T}}=K_{\mathrm{f}}^{2} / 2 v+\kappa_{\mathrm{f}}^{2} / 2 \alpha$, where $v^{\prime}=M_{\mathrm{P}}\left(M_{\mathrm{T}}+m_{\mathrm{e}}\right) / M, v=M_{\mathrm{T}}\left(M_{\mathrm{P}}+m_{\mathrm{e}}\right) / M$ are the corresponding reduced masses $(M=$ $M_{\mathrm{P}}+M_{\mathrm{T}}+m_{\mathrm{e}}$ with the masses $M_{\mathrm{P}}, M_{\mathrm{T}}$ and $m_{\mathrm{c}}$ of the projectile, target and electron, respectively), $\alpha$ and $\beta$ are defined in (3.5), and $\boldsymbol{\kappa}_{\mathrm{f}}$ is the momentum of the captured electron in the projectile frame of reference.

For the reduction of (3.2) it is convenient to introduce

$$
\begin{aligned}
& Q_{\mathrm{P}}=\alpha K_{\mathrm{f}}-K_{\mathrm{i}}^{\prime}-\boldsymbol{\kappa}_{\mathrm{f}} \quad \alpha=\frac{M_{\mathrm{P}}}{M_{\mathrm{P}}+m_{\mathrm{e}}} \\
& Q_{2}=\boldsymbol{K}_{\mathrm{f}}-\beta K_{\mathrm{i}}^{\prime}=Q_{\mathrm{P}}+\boldsymbol{v}+\boldsymbol{\kappa}_{\mathrm{f}} \quad \beta=\frac{M_{\mathrm{T}}}{M_{\mathrm{T}}+m_{\mathrm{e}}}
\end{aligned}
$$

where $\boldsymbol{v}=\boldsymbol{K}_{1}^{\prime} / \boldsymbol{v}^{\prime}$ is the collision velocity. In the second equality for $\boldsymbol{Q}_{2}$, small terms of the order of $m_{\mathrm{c}} / M_{\mathrm{P}}$ or $m_{\mathrm{c}} / M_{\mathrm{T}}$ have been disregarded. The quantity $Q_{1} \equiv Q_{\mathrm{P}}+\kappa_{\mathrm{f}}$ can be interpreted as the momentum transfer supplied by the projectile, while $Q_{2}=Q_{1}+v$ is the shifted momentum transfer as seen in the target frame of reference. Changing coordinates from $\boldsymbol{R}$ to $\boldsymbol{r}^{\prime}=\boldsymbol{R}+\alpha \boldsymbol{r}$, the two spatial integrals occurring in the first Born 
term $T_{1}$ can be separated in the following way:

$T_{1}=\int \mathrm{d} \boldsymbol{r} \exp \left[\mathrm{i}\left(\alpha \boldsymbol{K}_{\mathrm{f}}-\boldsymbol{K}_{\mathrm{i}}^{\prime}\right) \boldsymbol{r}\right] \phi_{\mathrm{f}}^{\mathrm{p}}(\boldsymbol{r}) \int \mathrm{d} \boldsymbol{r}^{\prime} \exp \left[-\mathrm{i}\left(\boldsymbol{K}_{\mathrm{f}}-\boldsymbol{\beta} \boldsymbol{K}_{\mathrm{i}}^{\prime}\right) \boldsymbol{r}^{\prime}\right] V_{\mathrm{T}}\left(\boldsymbol{r}^{\prime}\right) \phi_{\mathrm{i}}^{\mathrm{T}}\left(\boldsymbol{r}^{\prime}\right)$.

Taking for $\phi_{\mathrm{f}}^{\mathrm{P}}$ a Coulomb wave with momentum $\boldsymbol{\kappa}_{\mathrm{f}}$ and for $\phi_{\mathrm{i}}^{\mathrm{T}}$ a hydrogenic target is state, (3.6) is readily evaluated

$$
\begin{aligned}
& T_{1}=-2^{7 / 2} Z_{\mathrm{T}}^{3 / 2} Z_{\mathrm{P}} N(\eta)\left(\frac{1}{Z_{\mathrm{T}}^{2}+Q_{2}^{2}}+\frac{Z_{\mathrm{T}}-1}{\left(Z_{\mathrm{T}}+\lambda\right)^{2}+Q_{2}^{2}}\right) \frac{1}{Q_{\mathrm{P}}^{2}\left(Q_{\mathrm{P}}^{2}+2 \kappa_{\mathrm{r}} Q_{\mathrm{P}}\right)}\left(1+\frac{2 \kappa_{\mathrm{f}} Q_{\mathrm{P}}}{Q_{\mathrm{P}}^{2}}\right)^{-\mathrm{i} \eta} \\
& N(\eta)=\mathrm{e}^{\pi \eta / 2} \Gamma(1-\mathrm{i} \eta) \quad \eta=Z_{\mathrm{P}} / \kappa_{\mathrm{f}} .
\end{aligned}
$$

The second Born term $T_{2}$ has two contributions, $T_{2}^{(1)}$ and $T_{2}^{(2)}$, corresponding to the two parts of $V_{i}, V_{\mathrm{T}}^{\mathrm{S}}$ and $V_{\mathrm{P}}$, respectively. $T_{2}^{(\mathrm{I})}$ can again be evaluated analytically. To this end, the Fourier transforms of the quantities related to the target frame of reference, $V_{\mathrm{T}}$ and $V_{\mathrm{T}}^{\mathrm{S}} \phi_{\mathrm{i}}^{\mathrm{T}}$, are introduced such that the spatial integrals in the matrix elements of $V_{\mathrm{f}}$ and $V_{\mathrm{T}}^{\mathrm{S}}$ lead to four three-dimensional $\delta$-functions which fix the momenta of the Fourier transforms. One finds

$$
\begin{aligned}
& T_{2}^{(I)}=\int \mathrm{d} K^{\prime} \mathrm{d} \boldsymbol{k}^{\prime} \tilde{\phi}_{\mathrm{f}}^{\mathrm{p} *}\left(K^{\prime}-\alpha K_{\mathrm{f}}\right) \tilde{V}_{\mathrm{T}}\left(-k^{\prime}-\beta K^{\prime}+K_{\mathrm{f}}\right) \\
& \times \frac{1}{\left(K_{\mathrm{i}}^{\prime 2}-K^{\prime 2}\right) / 2 v^{\prime}+E_{\mathrm{i}}^{\mathrm{T}}-k^{\prime 2} / 2+\mathrm{i} \varepsilon} \\
& \times(\overbrace{\mathrm{T}}^{\mathrm{S}} \phi_{\mathrm{i}}^{\mathrm{T}})\left(\boldsymbol{k}^{\prime}-\beta\left(\boldsymbol{K}^{\prime}-\boldsymbol{K}_{\mathrm{i}}^{\prime}\right)\right)(2 \pi)^{3 / 2} \delta\left(\boldsymbol{K}^{\prime}-\boldsymbol{K}_{\mathrm{i}}^{\prime}\right)
\end{aligned}
$$

where the tilde denotes the Fourier transforms

$$
\begin{aligned}
& \tilde{V}_{\mathrm{T}}(q)=-\sqrt{\frac{2}{\pi}}\left(\frac{1}{q^{2}}+\frac{Z_{\mathrm{T}}-1}{\lambda^{2}+q^{2}}\right) \\
& (\overbrace{\mathrm{T}}^{\mathrm{s}} \phi_{1}^{\mathrm{T}})(q)=\frac{\sqrt{2}}{\pi} Z_{\mathrm{T}}^{3 / 2}\left(Z_{\mathrm{T}}-1\right)\left(\frac{1}{Z_{\mathrm{T}}^{2}+q^{2}}-\frac{1}{\left(Z_{\mathrm{T}}+\lambda\right)^{2}+q^{2}}\right) \\
& \tilde{\phi}_{\mathrm{f}}^{\mathrm{P}}(q)=\frac{N(\eta)}{\pi^{2}} \frac{1}{\left(q+\kappa_{\mathrm{f}}\right)^{2}\left(q^{2}-\kappa_{\mathrm{f}}^{2}\right)}\left(\frac{q^{2}-\kappa_{\mathrm{f}}^{2}-\mathrm{i} \varepsilon}{\left(q+\kappa_{\mathrm{f}}\right)^{2}}\right)^{-\mathrm{i} \eta} \quad \varepsilon \rightarrow 0 .
\end{aligned}
$$

The $K^{\prime}$ integral in (3.8) is trivial and the remaining three-dimensional integral can be evaluated with the help of the general formula (see Appendix)

$$
\begin{aligned}
I_{0} & \equiv \int \mathrm{d} k^{\prime} \frac{1}{k^{2}-k^{2}-\mathrm{i} \varepsilon} \frac{1}{\alpha^{2}+\left(k^{\prime}-q_{\mathrm{i}}\right)^{2}} \frac{1}{\beta^{2}+\left(k^{\prime}-q_{\mathrm{f}}\right)^{2}} \\
& =\frac{\mathrm{i} \pi^{2}}{2 \sqrt{\Delta}} \ln \left(\frac{a-\mathrm{i} \sqrt{\Delta}}{a+\mathrm{i} \sqrt{\Delta}}\right)^{2}
\end{aligned}
$$

with the definitions

$$
\begin{array}{lrr}
\Delta=k^{2} \gamma_{\mathrm{if}}^{2}+\gamma_{\mathrm{if}}\left(\gamma_{\mathrm{i}} \gamma_{\mathrm{f}}-4 \alpha \beta k^{2}\right)-\left(\alpha \gamma_{\mathrm{f}}+\beta \gamma_{\mathrm{i}}\right)^{2} & a=\alpha \gamma_{\mathrm{f}}+\beta \gamma_{\mathrm{i}}-\mathrm{i} k \gamma_{\mathrm{if}} \\
\gamma_{\mathrm{if}}=(\alpha+\beta)^{2}+\left(q_{\mathrm{f}}-q_{\mathrm{i}}\right)^{2} & \gamma_{\mathrm{i}}=\alpha^{2}+q_{\mathrm{i}}^{2}-k^{2} & \gamma_{\mathrm{f}}=\beta^{2}+q_{\mathrm{f}}^{2}-k^{2} .
\end{array}
$$


For the special case of (3.8) one has $k^{2}=2 E_{\mathrm{i}}^{\mathrm{T}}<0$ such that the parameter $a$ is positive with $0 \leqslant \sqrt{\Delta}<a$. This allows a reduction of $l_{0}$ to

$$
I_{0}(a>0)=\frac{2 \pi^{2}}{\sqrt{\Delta}} \arctan \frac{\sqrt{\Delta}}{a} .
$$

$T_{2}^{(1)}$ contains a sum of four integrals of the type (3.12) and is explicitly given by $T_{2}^{(1)}$

$$
\begin{aligned}
& =2^{9 / 2} Z_{\mathrm{T}}^{3 / 2}\left(Z_{\mathrm{T}}-1\right) Z_{\mathrm{P}} N(\eta) \frac{1}{Q_{2} Q_{\mathrm{P}}^{2}\left(Q_{\mathrm{P}}^{2}+2 \kappa_{\mathrm{r}} Q_{\mathrm{P}}\right)}\left(1+\frac{2 \kappa_{\mathrm{f}} Q_{\mathrm{P}}}{Q_{\mathrm{P}}^{2}}\right) \\
& \times \frac{1}{Z_{\mathrm{T}}^{2}+2 E_{\mathrm{i}}^{\mathrm{T}}}\left(\arctan \frac{\left(Z_{\mathrm{T}}^{2}+2 E_{\mathrm{i}}^{\mathrm{T}}\right) Q_{2}}{Z_{\mathrm{T}} \delta_{\mathrm{i}}+W_{\mathrm{i}}\left(Z_{\mathrm{T}}^{2}+Q_{2}^{2}\right)}\right.
\end{aligned}
$$$$
\left.+\left(Z_{\mathrm{T}}-1\right) \arctan \frac{\left(Z_{\mathrm{T}}^{2}+2 E_{\mathrm{i}}^{\mathrm{T}}\right) Q_{2}}{Z_{\mathrm{T}}\left(\lambda^{2}+\delta_{\mathrm{i}}\right)+\lambda\left(Z_{\mathrm{T}}^{2}-2 E_{\mathrm{i}}^{\mathrm{T}}\right)+W_{\mathrm{i}}\left[\left(Z_{\mathrm{T}}+\lambda\right)^{2}+Q_{2}^{2}\right]}\right)
$$$$
-\frac{1}{\left(Z_{\mathrm{T}}+\lambda\right)^{2}+2 E_{\mathrm{i}}^{\mathrm{T}}}\left(\arctan \frac{\left[\left(Z_{\mathrm{T}}+\lambda\right)^{2}+2 E_{\mathrm{i}}^{\mathrm{T}}\right] Q_{2}}{\left(Z_{\mathrm{T}}+\lambda\right) \delta_{\mathrm{i}}+W_{\mathrm{i}}\left[\left(Z_{\mathrm{T}}+\lambda\right)^{2}+Q_{2}^{2}\right]}\right.
$$

$$
\left.\left.-\left(Z_{\mathrm{T}}-1\right) \arctan \frac{\left[\left(Z_{\mathrm{T}}+\lambda\right)^{2}+2 E_{\mathrm{i}}^{\mathrm{T}}\right] Q_{2}}{\left(Z_{\mathrm{T}}+\lambda\right)\left(\lambda^{2}+\delta_{\mathrm{i}}\right)+\lambda\left[\left(Z_{\mathrm{T}}+\lambda\right)^{2}-2 E_{\mathrm{i}}^{\mathrm{T}}\right]+W_{\mathrm{i}}\left[\left(Z_{\mathrm{T}}+2 \lambda\right)^{2}+Q_{2}^{2}\right]}\right)\right]
$$

where $W_{\mathrm{i}}=\sqrt{-2 E_{\mathrm{i}}^{\mathrm{T}}}$ and $\delta_{\mathrm{i}}=Q_{2}^{2}-2 E_{\mathrm{i}}^{\mathrm{T}}$ have been introduced.

For the evaluation of the second part, $T_{2}^{(2)}$, similar techniques are employed. Again, the Fourier transforms of $\phi_{i}^{\mathrm{T}}$ and $V_{\mathrm{T}}$ are introduced in order to perform the spatial integration. The integration variables $K^{\prime}$ and $\boldsymbol{K}^{\prime}$ are transformed to new variables $T$ and $t$ by means of $T=K^{\prime}-\alpha K_{\mathrm{f}}$ and $t=k^{\prime}+\beta K^{\prime}-K_{\mathrm{f}}$ such that $T_{2}^{(2)}$ is written in the following form:

$$
\begin{aligned}
T_{2}^{(2)}=\int \mathrm{d} T \tilde{\phi}_{\mathrm{f}}^{\mathrm{P}}(T) \tilde{V}_{\mathrm{P}}\left(T+Q_{\mathrm{P}}+\kappa_{\mathrm{f}}\right) \\
\quad \times \int \mathrm{d} t \tilde{\phi}_{1}^{\mathrm{T}}\left(t+Q_{2}\right) \tilde{\mathrm{V}}_{\mathrm{T}}(t) \frac{1}{\kappa_{\mathrm{f}}^{2} / 2-v t-(T-t)^{2} / 2+\mathrm{i} \varepsilon} .
\end{aligned}
$$

The integral over $\boldsymbol{t}$ can be performed analytically by changing variables to $\boldsymbol{p}^{\prime}=\boldsymbol{t}-\boldsymbol{T}+\boldsymbol{v}$ which reduces it to the derivative of an integral of type $I_{0}$ from (3.10)

$$
\begin{aligned}
\int \mathrm{d} t \ldots=-4 & \left(\frac{Z_{\mathrm{T}}}{\pi}\right)^{3 / 2} \frac{\mathrm{d}}{\mathrm{d} Z_{\mathrm{T}}} \int \mathrm{d} p^{\prime} \frac{1}{p^{\prime 2}-k^{2}-\mathrm{i} \varepsilon} \frac{1}{Z_{\mathrm{T}}^{2}+\left(p^{\prime}-q_{\mathrm{i}}\right)^{2}} \\
& \times\left(\frac{1}{\left(p^{\prime}-q_{\mathrm{f}}\right)^{2}}+\frac{\mathrm{Z}_{\mathrm{T}}-1}{\lambda^{2}+\left(p^{\prime}-q_{\mathrm{f}}\right)^{2}}\right)
\end{aligned}
$$


with $k^{2}=v^{2}-2 v T+\kappa_{\mathrm{f}}^{2}, q_{\mathrm{i}}=v-T-Q_{2}$ and $q_{\mathrm{f}}=v-T$, such that $T_{2}^{(2)}$ reduces to a threedimensional integral

$$
\begin{aligned}
& T_{2}^{(2)}=\frac{-2^{5 / 2}}{\pi^{2}} Z_{\mathrm{T}}^{3 / 2} Z_{\mathrm{P}}^{2} N(\eta) \int \mathrm{d} T \frac{1}{\left(T+\kappa_{\mathrm{f}}\right)^{2}\left(T^{2}-\kappa_{\mathrm{f}}^{2}\right)}\left(\frac{T^{2}-\kappa_{\mathrm{f}}^{2}-\mathrm{i} \varepsilon}{\left(T+\kappa_{\mathrm{f}}\right)^{2}}\right)^{-\mathrm{i} \eta} \\
& \times \frac{1}{\left(T+Q_{\mathrm{P}}+\kappa_{\mathrm{f}}\right)^{2}}\left(\frac{\left(\partial / \partial Z_{\mathrm{T}}\right)\left(a_{1}^{2}+\Delta_{1}\right)}{a_{1}\left(a_{1}^{2}+\Delta_{1}\right)}-\frac{1}{\Delta_{\mathrm{l}}} \frac{\partial \Delta_{\mathrm{l}}}{\partial Z_{\mathrm{T}}}\left[\frac{1}{a_{1}}-\frac{\mathrm{i}}{4 \sqrt{\Delta_{1}}} \ln \left(\frac{a_{1}-\mathrm{i} \sqrt{\Delta_{\mathrm{f}}}}{a_{1}+\mathrm{i} \sqrt{\Delta_{\mathrm{l}}}}\right)^{2}\right]\right. \\
&\left.+\left(Z_{\mathrm{T}}-1\right)\left\{\frac{\left(\partial / \partial Z_{\mathrm{T}}\right)\left(a_{2}^{2}+\Delta_{2}\right)}{a_{2}\left(a_{2}^{2}+\Delta_{2}\right)}-\frac{1}{\Delta_{2}} \frac{\partial \Delta_{2}}{\partial Z_{\mathrm{T}}}\left[\frac{1}{a_{2}}-\frac{\mathrm{i}}{4 \sqrt{\Delta_{2}}} \ln \left(\frac{a_{2}-\mathrm{i} \sqrt{\Delta_{2}}}{a_{2}+\mathrm{i} \sqrt{\Delta_{2}}}\right)^{2}\right]\right\}\right)
\end{aligned}
$$

with the definitions

$$
\begin{aligned}
& a_{1}=Z_{\mathrm{T}} \gamma_{\mathrm{f}}^{\left(\frac{1}{2}\right)}+\lambda_{1} \gamma_{\mathrm{i}}^{\left(\frac{1}{2}\right)}-\mathrm{i} k \gamma_{\mathrm{if}}^{\left(\frac{1}{2}\right)} \quad \lambda_{\mathrm{I}}=0, \lambda_{2}=\lambda \\
& \Delta_{1}=k^{2}\left(\gamma_{i f}^{(1)}\right)^{2}+\gamma_{i f}^{(2)}\left(\gamma_{i}^{\left(\frac{1}{2}\right)} \gamma_{\mathrm{f}}^{\left(\frac{1}{2}\right)}-4 Z_{\mathrm{T}} \lambda_{2} k^{2}\right)-\left(Z_{\mathrm{T}} \gamma_{\mathrm{f}}^{(2)}+\lambda_{1} \gamma_{\mathrm{i}}^{\left(\frac{1}{2}\right)}\right)^{2} \\
& \gamma_{\mathrm{i}}^{(1)}=\gamma_{\mathrm{i}}^{(2)}=Z_{\mathrm{T}}^{2}+q_{\mathrm{i}}^{2}-k^{2} \quad \gamma_{\mathrm{f}}^{(1)}=\lambda_{\mathrm{I}}^{2}+q_{\mathrm{f}}^{2}-k^{2} \quad \gamma_{\mathrm{if}}^{(2)}=\left(Z_{\mathrm{T}}+\lambda_{1}\right)^{2}+Q_{2}^{2} .
\end{aligned}
$$

This integral has to be evaluated numerically. When doing that, care has to be taken of the singularities of the integrand at $T=-\kappa_{\mathrm{f}}$ and $T=-\boldsymbol{Q}_{\mathrm{P}}-\boldsymbol{\kappa}_{\mathrm{f}}$.

From the transition matrix element, the differential cross section for the emission of an electron with momentum $k_{\mathrm{f}}$ (in the target frame of reference) is obtained by

$$
\frac{\mathrm{d} \sigma^{\mathrm{B} 2}}{\mathrm{~d} k_{\mathrm{f}}}=\frac{1}{4 \pi^{2} v} \int \mathrm{d} K_{\mathrm{f}}\left|T_{\mathrm{fi}}\right|^{2} \delta\left(E_{\mathrm{i}}^{\mathrm{tot}}-E_{\mathrm{f}}^{\mathrm{tot}}\right) \text {. }
$$

Replacing $K_{f}$ by the momentum transfer $Q_{P}$ with the help of (3.5), such that the argument of the $\delta$-function changes into $E_{\mathrm{f}}^{\text {tot }}-E_{\mathrm{i}}^{\text {tot }}=k_{\mathrm{f}}^{2} / 2-E_{\mathrm{i}}^{\mathrm{T}}+Q_{\mathrm{p}} v$, the doubly differential cross section for electron ejection into the solid angle $d \Omega_{\mathrm{f}}$ reduces to

$$
\begin{aligned}
& \frac{\mathrm{d}^{2} \sigma^{\mathrm{B} 2}}{\mathrm{~d} E_{\mathrm{f}} \mathrm{d} \Omega_{\mathrm{f}}}=\frac{k_{\mathrm{f}} N_{\mathrm{i}}}{2 \pi^{2} v^{2}} \int_{0}^{\pi} \mathrm{d} \varphi_{Q} \int_{Q_{\min }}^{\infty} Q_{\mathrm{P}} \mathrm{d} Q_{\mathrm{P}}\left|T_{\mathrm{l}}+T_{2}^{(1)}+T_{2}^{(2)}\right|^{2} \\
& \mathrm{Q}_{\min }=\left(k_{\mathrm{f}}^{2} / 2-E_{\mathrm{i}}^{\mathrm{T}}\right) / v
\end{aligned}
$$

where $E_{\mathrm{f}}=k_{\mathrm{f}}^{2} / 2$ and $N_{\mathrm{i}}$ is the number of the target K-shell electrons. Spherical coordinates $Q_{\mathrm{P}}, \theta_{Q}$ and $\varphi_{Q}$ have been chosen for $Q_{\mathrm{P}}$, with $\cos \theta_{Q}=-Q_{\min } / Q_{\mathrm{P}}$ determined by the energy-conserving $\delta$-function. We should like to note that, apart from the perturbative treatment of the screening potential $V_{T}^{\mathrm{S}}$ in the initial channel, (3.19) is exact within the second-order Born prescription.

\section{Impulse approximation}

The impulse approximation for electron capture is a theory which goes beyond the second-order Born approximation by treating one of the potentials $\left(V_{\mathrm{p}}, V_{\mathrm{T}}\right)$ exactly and the other one in first order. This theory is therefore suited to asymmetric collision systems and exists in two forms, the prior (for $Z_{\mathrm{P}} \ll Z_{T}$ ) and the post (for $Z_{\mathrm{P}} \gg Z_{\mathrm{T}}$ ). At very large collision velocities, the IA reduces to the second Born theory. The transition 
amplitude in the prior IA is given by Briggs (1977)

$$
a_{\mathrm{f}}^{\mathrm{A}, \mathrm{p} \text { rior }}=-\mathrm{i} \int_{-\infty}^{\infty} \mathrm{d} t \int \mathrm{d} \boldsymbol{q}\left\langle\phi_{\mathrm{f}}^{\mathrm{P}} \mid q\right\rangle\left\langle\phi_{\mathrm{q}}^{\mathrm{T}}\left|V_{\mathrm{P}}\right| \phi_{\mathrm{i}}^{\mathrm{T}}\right\rangle
$$

which implies that the capture process from the bound target state $\phi_{i}^{\top}$ to the final projectile state $\phi_{f}^{\mathrm{P}}$ proceeds via an intermediate continuum target eigenstate $\phi_{q}^{\mathrm{T}}$ of momentum $q$. The state $|q\rangle$ describes a plane wave, $(2 \pi)^{-3 / 2} \exp (\mathrm{i} q \boldsymbol{r})$, where $\boldsymbol{r}$ is the electronic coordinate in the target frame of reference. The introduction of a full peaking approximation in the ionization matrix element $\left\langle\phi_{q}^{\mathrm{T}}\left|V_{\mathrm{P}}\right| \phi_{\mathrm{i}}^{\mathrm{T}}\right)$ leads to a simple factorization of the capture cross section into the doubly differential cross section $\mathrm{d}^{2} \sigma^{\mathrm{t}} / \mathrm{d} E_{\mathrm{f}} \mathrm{d} \Omega_{\mathrm{f}}$ for the ejection of a target electron with energy $E_{\mathrm{f}}=k_{\mathrm{f}}^{2} / 2$ into the solid angle $\mathrm{d} \Omega_{\mathrm{f}}$, times the absolute squared normalization constant $N(\eta)$ of the projectile Coulomb wave (Jakubassa-Amundsen 1988)

$$
\begin{aligned}
\frac{\mathrm{d}^{2} \sigma^{\mathrm{IA}, \text { prior }}}{\mathrm{d} E_{\mathrm{f}} \mathrm{d} \Omega_{\mathrm{f}}}= & |N(\eta)|^{2} \frac{\mathrm{d}^{2} \sigma^{\mathrm{I}}}{\mathrm{d} E_{\mathrm{f}} \mathrm{d} \Omega_{\mathrm{f}}} \\
& =\frac{2 \pi \eta}{1-\mathrm{e}^{-2 \pi \eta}} \frac{4 Z_{\mathrm{P}}^{2} k_{\mathrm{f}}}{v} N_{\mathrm{i}} \int \mathrm{d} q \frac{1}{q^{4}}\left|\left\langle\phi_{k_{\mathrm{f}}}^{\mathrm{T}}\left(\boldsymbol{r}^{\prime}\right)\left|\mathrm{e}^{-\mathrm{i} q r^{\prime}}\right| \phi_{\mathrm{i}}^{\mathrm{T}}\left(\boldsymbol{r}^{\prime}\right)\right\rangle\right|^{2} \delta\left(E_{\mathrm{f}}-E_{\mathrm{i}}^{\mathrm{T}}+q v\right)
\end{aligned}
$$

where $E_{1}^{\top}$ is the initial-state energy, $N$, the occupation number of the initial subshell, and $\eta=Z_{\mathrm{P}} /\left|\boldsymbol{k}_{\mathrm{f}}-\boldsymbol{v}\right|$. The peaked IA accounts well for absolute intensities, but due to the peaking approximation involved in (4.2), the information about the cusp asymmetry is lost. However, in the region of validity of this approximation $\left(Z_{\mathrm{P}} \ll Z_{\mathrm{T}}, v>Z_{\mathrm{T}}\right.$ or $Z_{P}\left\langle Z_{T}, v \gg Z_{T}\right.$ ) the asymmetry is small anyhow, as can be shown by means of the less restrictive transverse peaking approximation (Jakubassa-Amundsen 1988).

The post form of the IA transition amplitude differs from (4.1) by the presence of an intermediate continuum projectile state $\phi_{q}^{\mathrm{P}}$ (instead of a target state)

$$
a_{\mathrm{fi}}^{\mathrm{IA}, \text { post }}=-\mathrm{i} \int_{-\infty}^{\infty} \mathrm{d} t \int \mathrm{d} q\left\langle\phi_{\mathrm{f}}^{\mathrm{P}}\left|V_{\mathrm{T}}\right| \phi_{q}^{\mathrm{P}}\right\rangle\left\langle q \mid \phi_{\mathrm{l}}^{\mathrm{T}}\right\rangle .
$$

This form contains a continuum-continuum scattering matrix element which makes the full peaking approximation diverge. Hence, the transverse peaking approximation (which affects the two dimensions perpendicular to $v$ ) is made in the scattering matrix element. This means that an extra integral occurs in the transition amplitude as compared to the full-peaking result, and the doubly differential capture cross section has to be calculated from

$$
\begin{aligned}
& \frac{\mathrm{d}^{2} \sigma^{\mathrm{IA}, \text { post }}}{\mathrm{d} E_{\mathrm{f}} \mathrm{d} \Omega_{\mathrm{f}}}=\frac{2 \pi k_{\mathrm{f}}}{v} N_{\mathrm{i}} \int \mathrm{d} q \delta\left(E_{\mathrm{f}}-E_{\mathrm{s}}^{\mathrm{T}}+q v\right) \\
& \times\left|\int_{-\infty}^{\infty} \mathrm{d} s_{z} f_{0}\left(q, s_{z}\right)\left\langle\phi_{\mathrm{f}}^{\mathrm{P}}\left|\exp \left[\mathrm{I}\left(q+k_{\mathrm{f}}-v-s_{z} e_{\mathrm{z}}\right) \boldsymbol{r}\right]\right| \phi_{s_{z} e_{\mathrm{z}}}^{\mathrm{P}}\right\rangle\right|^{2}
\end{aligned}
$$

$f_{0}\left(q, s_{z}\right)=\int \mathrm{d} s_{\perp} \tilde{\phi}_{\mathrm{i}}^{\mathrm{\Upsilon}}(s+v) \tilde{V}_{\mathrm{T}}\left(\boldsymbol{q}+\boldsymbol{k}_{\mathrm{f}}-\boldsymbol{v}-s\right)$

with $e_{z}=v / v$. The transition matrix element $\left\langle\phi_{f}^{\mathrm{P}}\left|\exp \left(\mathrm{i} Q_{r}\right)\right| \phi_{s_{s} e_{\mathrm{s}}}^{\mathrm{P}}\right\rangle$ and the convolution integral $f_{0}\left(q, s_{z}\right)$ of the Fourier-transformed initial state $\tilde{\phi}_{i}^{\mathrm{T}}$ and target potential $\tilde{V}_{\mathrm{T}}$ can be evaluated analytically for hydrogenic wavefunctions (Jakubassa-Amundsen 1983). 
For the target potential (i.e. the interaction between the active electron and the target core) the following approximation has been used

$$
V_{\mathrm{T}}\left(r^{\prime}\right)=-\frac{Z_{\mathrm{T}}-1}{r^{\prime}} \mathrm{e}^{-r / d}\left(1+\frac{r^{\prime}}{2 d}\right)-\frac{1}{r^{\prime}}
$$

where the parameter $d=0.234$ is adjusted to reproduce the experimental ground state binding energy. This potential should be slightly superior to the one used in the second Born approximation, equations (3.1) with (3.2).

\section{Experimental cusp intensities in comparison with theory}

In order to investigate the dependence on the collision velocity and on the projectile species, cusp spectra for $1.25 \mathrm{MeV}(v=7.08 \mathrm{au}), 2.5 \mathrm{MeV}(v=10 \mathrm{au})$ and $4.2 \mathrm{MeV}(v=$ $12.97 \mathrm{au}) \mathrm{H}^{+}+\mathrm{Ne}$ and $25 \mathrm{MeV}(v=7.91 \mathrm{au}), 40 \mathrm{MeV}(v=10 \mathrm{au})$ and $80 \mathrm{MeV}(v=$ $14.15 \mathrm{au}) \mathrm{O}^{8+}+\mathrm{Ne}$ have been recorded at fixed detector resolution. Our results for proton impact are shown in figure 3 and for oxygen impact in figure 4 . The background has been subtracted with the help of separately recorded background spectra. Since the background spectra vary smoothly across the cusp region, it was in most cases sufficient to subtract a constant or linear momentum-dependent background. For the proton case, the cusp spectra are nearly symmetric, whereas for oxygen impact they are strongly skewed to the low-energy side. In all cases, the intensity decreases strongly with collision velocity.

The theoretical calculations have been performed within the second-order Born approximation (3.19) and the impulse approximation. Experimental initial-state binding energies have been used in all calculations, together with Slater-screened hydrogenic target functions in case of IA and with unscreened target functions in case of B2. For the asymmetric collision system $\mathrm{H}^{+}+\mathrm{Ne}$, results are given for the B2 theory (including only capture from the target $\mathrm{K}$ shell) and for the prior impulse approximation (4.2). Due to the factorization of the capture cross section in the prior IA, we have found it easy to include capture from the target $\mathrm{L}$ shell. At the lowest impact energy $(1.25 \mathrm{MeV})$ the $\mathrm{L}$-shell electrons give the dominant contribution, the doubly differential cross section ratio $R_{\mathrm{KL}}=\mathrm{d}^{2} \sigma / \mathrm{d} E_{\mathrm{f}} \mathrm{d} \Omega_{\mathrm{f}}(K+L) / \mathrm{d}^{2} \sigma / \mathrm{d} E_{\mathrm{f}} \mathrm{d} \Omega_{\mathrm{f}}(K)$ in the peak maximum being as large as $R_{\mathrm{KL}}=6.55$. It decreases with energy ( $R_{\mathrm{KL}}=1.89$ for $2.5 \mathrm{MeV}$ and 1.28 for $4.2 \mathrm{MeV}$ ). Since the peak is only weakly asymmetric, the shape depends very little on the initial target state.

In order to facilitate comparison with experiment, all theories have been normalized to experiment in the peak maximum. As is obvious from figure 3 , the $\mathrm{B} 2$ and the $\mathrm{IA}$ theories give very similar results for the peak shape. For the higher impact energies they reproduce the experimental shape quite well. Also for $1.25 \mathrm{MeV}$ protons, theory should give a correct account for the peak width, as follows from a comparison with the early $p+\mathrm{Ne}$ experiments (Cranage and Lucas 1976, Rødbro and Andersen 1979) if the $v \theta_{0}$ scaling of the width (equation (6.6)) is applied. The large width measured in our $\mathrm{H}^{+}+\mathrm{Ne}$ experiments at the lowest impact energy is presumably an artefact, because at the Heidelberg facilities no negative bias had been applied to the detector. Hence low-energy stray electrons which result from the beam hitting the apparatus could not be prevented from entering the detector. At our higher impact energies, the contribution of these electrons becomes insignificant. 

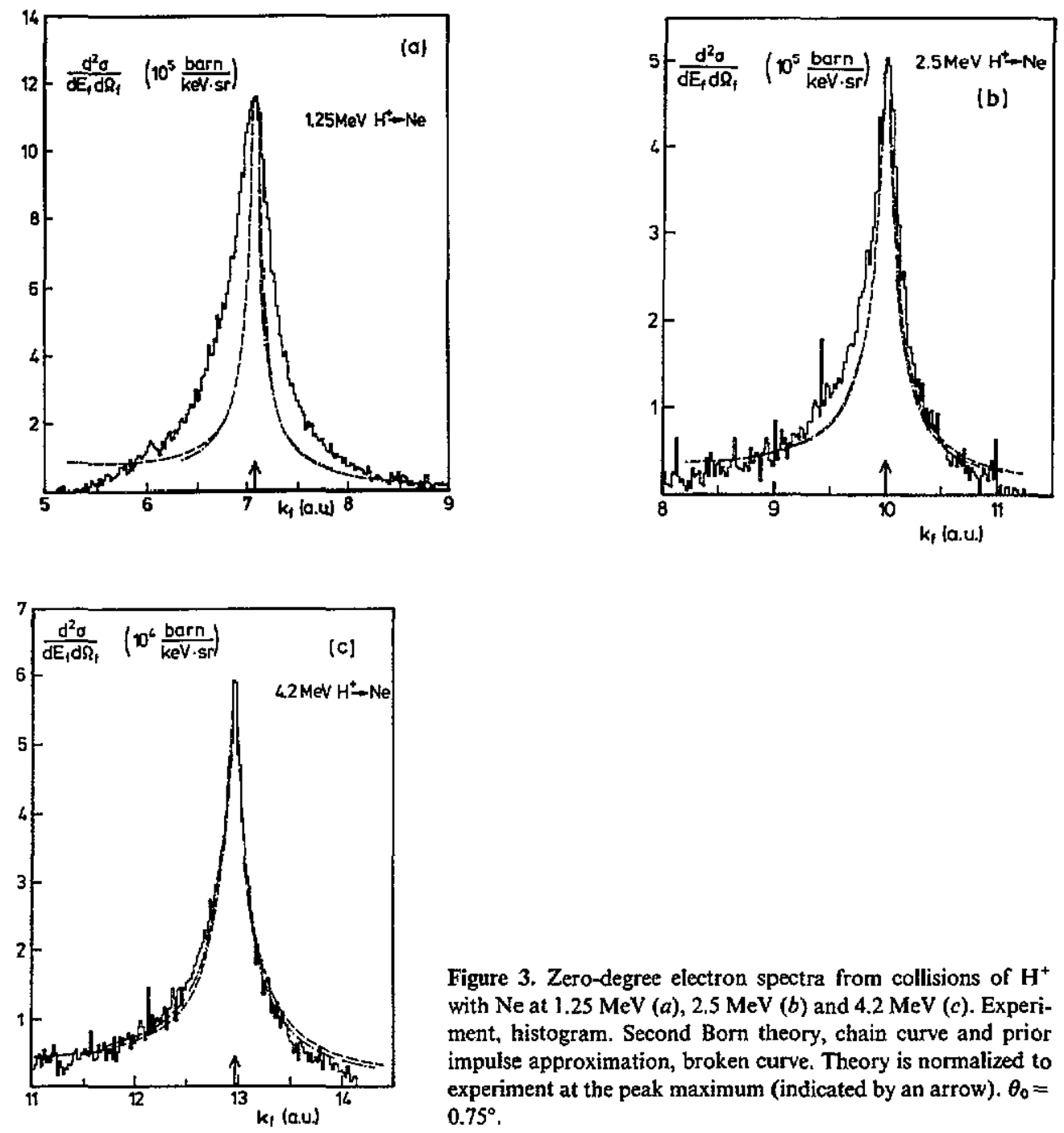

Figure 3. Zero-degree electron spectra from collisions of $\mathbf{H}^{+}$ with $\mathrm{Ne}$ at $1.25 \mathrm{MeV}(a), 2.5 \mathrm{MeV}(b)$ and $4.2 \mathrm{MeV}(c)$. Experiment, histogram. Second Born theory, chain curve and prior impulse approximation, broken curve. Theory is normalized to experiment at the peak maximum (indicated by an arrow). $\theta_{0}=$ $0.75^{\circ}$.

The failure of theory to describe experiments at collision velocities below $v=Z_{\mathrm{T}}$ is, however, apparent when the absolute values are compared (table 1). While for $v \geqslant 10$ au the IA can reproduce the measured intensity within the experimental absolute uncertainty of $\sim 40 \%$, the IA drastically overestimates the experimental yield at lower velocities. The reason is not only the invalidity of the full peaking approximation (being a high-energy approximation), but also the breakdown of the IA itself. The overestimation of experiment by theory is even worse for the second Born theory (taking into consideration that the contribution from the L-shell electrons is omitted). The reason may be that the symmetric $\mathbf{B} 2$ theory needs a higher velocity to be applicable than the asymmetric IA for a system with $Z_{\mathrm{P}} / Z_{\mathrm{T}}=0.1$.

For the near-symmetric collision system $\mathrm{O}^{8+}+\mathrm{Ne}$ no $\mathrm{B} 2$ calculations are available which account for target screening (which decreases the absolute cross section considerably). Moreover, neither of the two IA versions is expected to work since we have the unfortunate situation that $Z_{\mathrm{P}} \approx Z_{\mathrm{T}} \approx v$. Therefore, we present only tentative results for 

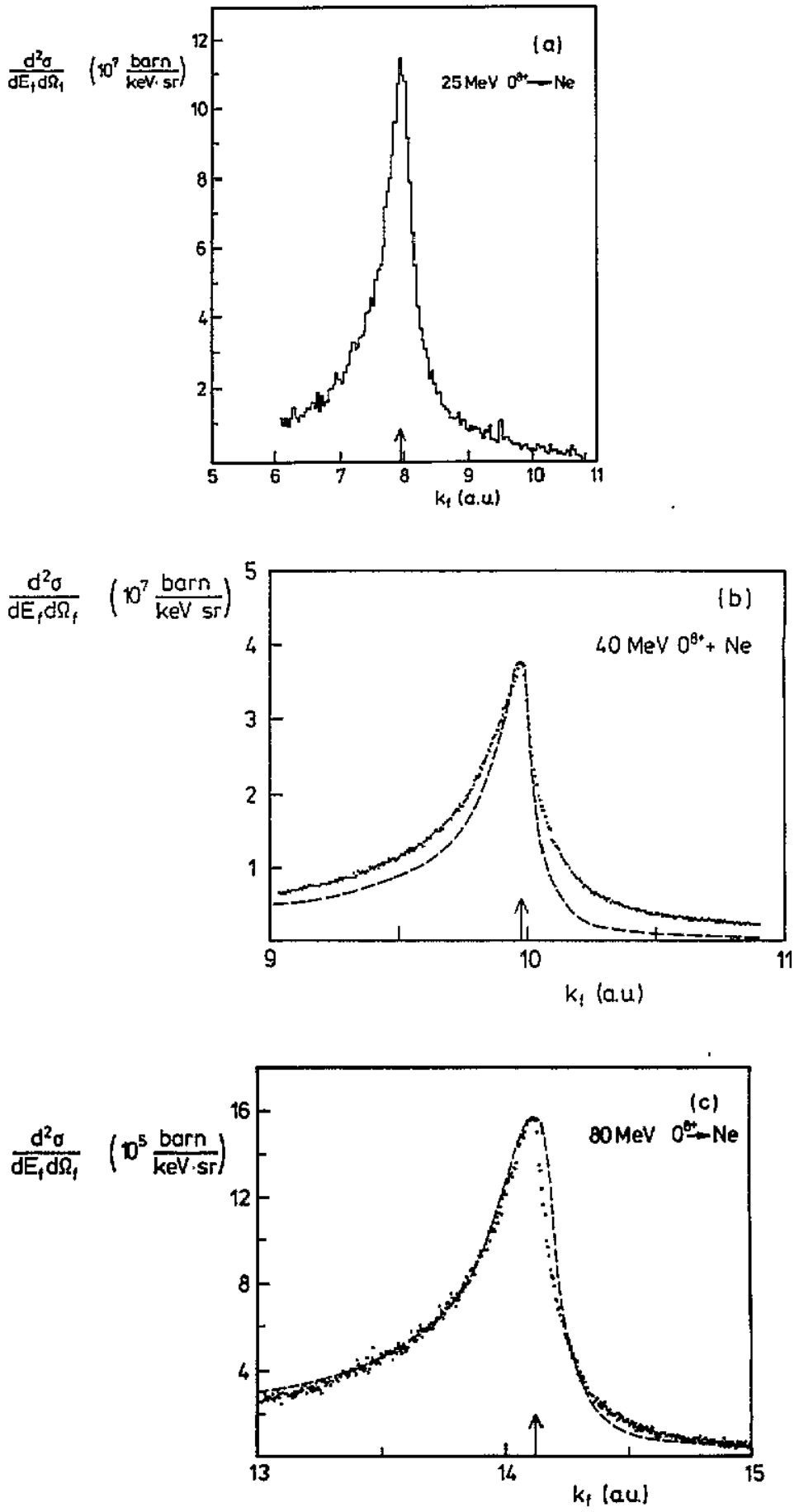

Figure 4. Zero-degree electron spectra from collisions of $\mathrm{O}^{8 \mathrm{r}}$ with $\mathrm{Ne}$ at $25 \mathrm{MeV}(a)$, $40 \mathrm{MeV}(b)$ and $80 \mathrm{MeV}(c)$. Experiment, histogram $(a)$ and dots $((b)$ and $(c))$. Post iA, broken curve. Theory is normalized to experiment at the peak maximum (indicated by an arrow). $\theta_{0}=0.8^{\circ}$ except for $25 \mathrm{MeV}$ where it is $0.75^{\circ}$. 
Table 1. Ratio $F=\mathrm{d}^{2} \sigma_{\mathrm{exp}} / \mathrm{d}^{2} \sigma_{\text {theor }}$ for a Ne target, where $\mathrm{d}^{2} \sigma \equiv\left\langle\mathrm{d}^{2} \sigma / \mathrm{d} E_{\mathrm{f}} \mathrm{d} \Omega_{\mathrm{f}}\right\rangle_{\theta_{\mathrm{Q}} \Delta E}$. The second Born approximation (B2) and the post i $\mathrm{A}$ is for $\mathrm{K}$-shell capture only, while the prior IA includes capture from all target shells.

\begin{tabular}{llllll}
\hline Projectile & $\begin{array}{l}\text { Energy } \\
(\mathrm{MeV})\end{array}$ & $F^{\mathrm{g} 2}$ & $F^{\text {post IA }}$ & $F^{\text {proriA }}$ & $\theta_{0}(\mathrm{deg})$ \\
\hline $\mathrm{H}^{+}$ & 1.25 & 0.304 & & 0.143 & 0.75 \\
& 2.5 & 0.345 & & 0.962 & 0.75 \\
& 4.2 & 0.159 & & 0.775 & 0.75 \\
$\mathrm{O}^{8+}$ & 40 & & 0.320 & & 0.8 \\
& 80 & & 0.0914 & & 0.8 \\
\hline
\end{tabular}

the post IA (4.4) at the highest velocities. Because of the less restrictive peaking approximation inherent in (4.4), as compared to the prior IA, the post IA is superior in describing the cusp shape, whereas both IA versions provide similar peak intensities. From figure 4 it follows that the experimental peak shape is reasonably well reproduced by the post IA. However, the experimental intensity is strongly overpredicted (table 1). Only capture from the $\mathrm{K}$ shell has been considered but we have estimated the contribution from the $\mathrm{L}$ shell electrons with the help of the prior IA. We have found that this contribution is small at $80 \mathrm{MeV}\left(R_{\mathrm{KL}}=1.2\right)$, but important at $40 \mathrm{MeV}\left(R_{\mathrm{KL}}=1.89\right)$.

\section{Shape parameters of the forward peak for variable angular resolution}

According to the behaviour of the normalization constant $N(\eta)$ of the final-state Coulomb wave $\phi_{\mathrm{f}}^{\mathrm{P}}$ the doubly differential cross section for electron emission diverges in the limit $\kappa_{\mathrm{f}}=\left|\boldsymbol{k}_{\mathrm{f}}-\boldsymbol{v}\right| \rightarrow 0$

$$
\frac{\mathrm{d}^{2} \sigma}{\mathrm{d} E_{\mathrm{f}} \mathrm{d} \Omega_{\mathrm{f}}} \sim|N(\eta)|^{2} \approx 2 \pi \eta \quad \eta=Z_{\mathrm{p}} / \kappa_{\mathrm{f}} \rightarrow \infty
$$

In an experimental situation the angular resolution $\theta_{0}$ and energy resolution $\Delta E$ of the detector make it necessary to average (6.1) over $\theta_{0}$ and $\Delta E$ such that the peak intensity becomes finite. However, both peak intensity and peak width will then strongly depend on the detector resolution (Dettmann et al 1974). Mecbach et al (1981) have suggested a procedure of data reduction which should eliminate this detector dependence and hence allow for a comparison of data on the cusp shape which are obtained under different experimental conditions. To this end, the differential cross section is transformed into the projectile frame of reference and subsequently expanded around $\kappa_{\mathrm{f}}=$ 0 in terms of Legendre polynomials $P_{2}$ (Macek et al 1981).

$$
\frac{\mathrm{d}^{2} \sigma}{\mathrm{d} \varepsilon_{\mathrm{f}} \mathrm{d} \Omega_{\mathrm{f}}^{\prime}}=\frac{\kappa_{\mathrm{f}}}{k_{\mathrm{f}}} \frac{\mathrm{d}^{2} \sigma}{\mathrm{d} E_{\mathrm{f}} \mathrm{d} \Omega_{\mathrm{f}}}=\sum_{n t} B_{n i} K_{\mathrm{f}}^{n} P_{l}\left(\cos \theta_{\mathrm{f}}^{\prime}\right)
$$

where $\varepsilon_{\mathrm{f}}=\kappa_{\mathrm{f}}^{2} / 2$ and the emission angle $\theta_{\mathrm{f}}^{\prime}$ in the projectile frame of reference is defined by $\cos \theta_{\mathrm{f}}^{\prime}=\left(k_{\mathrm{f}} v / v-v\right) / \kappa_{\mathrm{f}}$. Theoretically, for a given power of $n$, the coefficients $B_{n l}$ are readily obtained by inverting $(6.2)$

$$
B_{n l}=\left.\frac{2 l+1}{2 n !} \int_{-1}^{l} \mathrm{~d}\left(\cos \theta_{\mathrm{f}}^{\prime}\right) P_{l}\left(\cos \theta_{\mathrm{f}}^{\prime}\right) \frac{\mathrm{d}^{n}}{\mathrm{~d} \kappa_{\mathrm{f}}^{n}}\left(\frac{\mathrm{d}^{2} \sigma}{\mathrm{d} \varepsilon_{\mathrm{f}} \mathrm{d} \Omega_{\mathrm{f}}^{\prime}}\right)\right|_{\kappa_{\mathrm{f}}=0}
$$


where it is indicated that the derivatives have to be taken at $\kappa_{\mathrm{f}}=0$. For an experimental determination of the shape parameters $B_{n t}$ the series (6.2) is truncated to (at most) six terms, $l \leqslant 2$ and $n \leqslant 1$. The target-frame cross section $\mathrm{d}^{2} \sigma / \mathrm{d} E_{\mathrm{f}} \mathrm{d} \Omega_{\mathrm{f}}$ from (6.2) is folded with the transmission function of the spectrometer and the resulting expression is fitted to the experimental spectra.

From theoretical considerations a series expansion in the emission angle $\theta_{f}^{f}$ does, however, not formally exist for $\kappa_{\mathrm{f}}=0$ in the case of electron capture. This is due to the non-analytic behaviour of the Coulomb eigenfunction which is easily seen when the Fourier-transformed Coulomb wave $\tilde{\phi}_{\mathrm{f}}^{\mathrm{P}}$ is considered in the limit $\kappa_{\mathrm{f}} \rightarrow 0$ (cf (3.9))

$$
\begin{aligned}
& {\phi_{f}^{\mathrm{P}^{*}}}_{(q)} \rightarrow \frac{Z_{\mathrm{P}}}{\pi^{2}} N(\eta) \frac{1}{q^{4}} \exp \left(-2 \mathrm{i} Z_{\mathrm{P}} \cos \theta_{q, \mu_{\mathrm{c}}} / q\right) \quad \kappa_{\mathrm{f}} \rightarrow 0 \\
& \cos \theta_{q, \kappa_{\mathrm{f}}}=\cos \theta_{q} \cos \theta_{\mathrm{f}}^{\prime}+\sin \theta_{q} \sin \theta_{\mathrm{f}}^{\prime} \cos \varphi
\end{aligned}
$$

where the phase factor depends on the direction $\left(\theta_{f}^{\prime}\right)$ of the momentum $\kappa_{f}$ even for $\kappa_{\mathrm{f}}=$ 0 . This phase is different for $\theta_{\mathrm{f}}^{\prime}=0^{\circ}$ and $180^{\circ}$, and thus leads to an asymmetric cusp with an enhanced intensity on the low-energy wing in every higher-order theory. Moreover, its $\theta_{f}^{\prime}$ dependence is non-analytic (in contrast to electron loss) since according to (3.14) the momentum $q$ in (6.4) can become zero. Note that in a first-order theory or in the fully peaked prior IA, the phase from the Coulomb wave $\phi_{f}^{\mathrm{P}}$ drops out when the transition probability is calculated and hence the resulting cusp is symmetric. As a consequence of this non-analyticity, the fit of a truncated series (6.2) to the experimental spectra will provide shape parameters which are not independent of the detector resolution and which therefore are inadequate for a unified comparison of data recorded with different experimental set-ups (Berényi et al 1987, Lucas and Steckelmacher 1987).

In order to demonstrate that the $B_{n t}$ parameters indeed depend on the detector resolution, the cusp spectra from $40 \mathrm{MeV}$ and $80 \mathrm{MeV} \mathrm{O}^{8+}$ on Ne have been recorded at six different angular resolutions, ranging from $\theta_{0}=0.1^{\circ}$ to $1.2^{\circ}$. A selection of these spectra is depicted in figures 5 and 6 . The spectra are plotted on a relative scale, but absolute values may readily be obtained from figure 4 by means of scaling the cusp maximum with $\theta_{0}^{-1}$ which is rather well satisfied both experimentally and theoretically. Each spectrum has been fitted separately with the series (6.2) truncated to six terms. This has been done by integrating (6.2) over the experimental angular and momentum resolution

$$
\begin{aligned}
I\left(k_{\mathrm{f}}, \theta_{0}\right)= & \int_{k_{\mathrm{r}}(1-\Delta / 2)}^{k_{\mathrm{f}}(1+\Delta / 2)} \mathrm{d} k_{\mathrm{f}} \int_{0}^{\theta_{0}} \sin \theta_{\mathrm{f}} \mathrm{d} \theta_{\mathrm{f}} \frac{\mathrm{d}^{2} \sigma}{\mathrm{d} k_{\mathrm{f}} \mathrm{d} \Omega_{\mathrm{f}}} \\
& =\sum_{n=0}^{1} \sum_{l=0}^{2} B_{n l} \int_{k_{\mathrm{f}}(1-\Delta / 2)}^{k_{\mathrm{f}}(1+\Delta / 2)} k_{\mathrm{f}}^{2} \mathrm{~d} k_{\mathrm{f}} \int_{0}^{\theta_{0}} \sin \theta_{\mathrm{f}} \mathrm{d} \theta_{\mathrm{f}} \kappa_{\mathrm{f}}^{n-1} P_{l}\left(\cos \theta_{\mathrm{f}}^{\prime}\right)
\end{aligned}
$$

where $\theta_{\mathrm{f}}$ is the target frame ejection angle of the electron and $\Delta=0.0025$. The step-like resolution function is consistent with our procedure for determining $\theta_{0}$ and $\Delta$. The angular integration can be performed analytically, while the $k_{\mathrm{f}}$ integration is done numerically. By fitting (6.5) to the experimental spectra, the $B_{n l}$ have been determined for each of the six values of $\theta_{0}$. We have, however, not found it possible to obtain a satisfactory fit to all spectra with only one set of $B_{n t}$. As an example, figure 5(a) shows the $0.4^{\circ}$ spectrum for $40 \mathrm{MeV}$ impact together with the fit with $B_{n l}\left(0.4^{\circ}\right)$. This fit is hardly distinguishable from the experimental data. If, however, the $B_{n l}\left(0.4^{\circ}\right)$ are inserted 


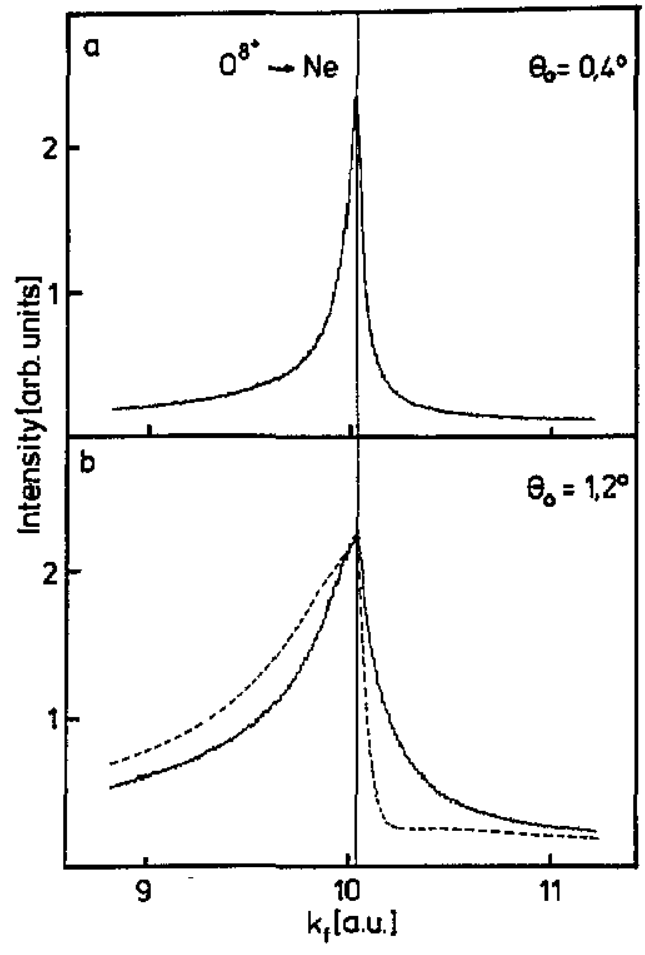

Figure 5. Cusp electron spectra from collisions of $40 \mathrm{MeV} \mathrm{O}^{8+}$ with $\mathrm{Ne}$ at $\theta_{0}=0.4^{\circ}(a)$ and $1.2^{\circ}(b)$. Experiment, histogram. Theory, broken curve: $(a)$ $B_{n l}$ fit to the $0.4^{\circ}$ spectrum, (b) constructed spectrum using the shape parameters from the $0.4^{\circ} \mathrm{fit}$.

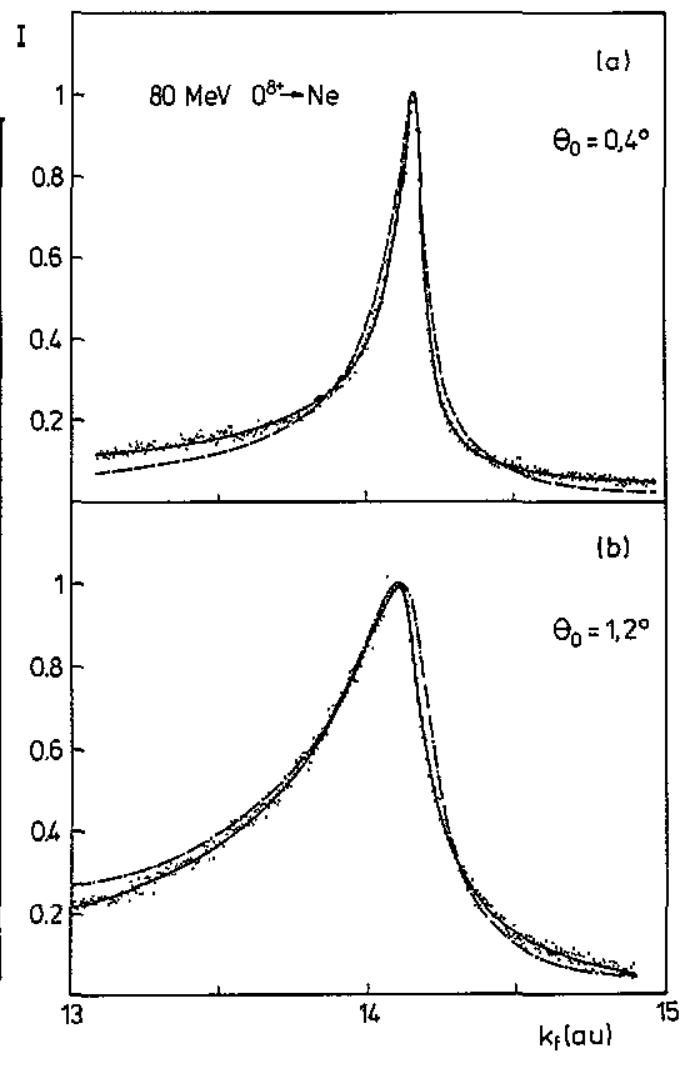

Figure 6. Cusp electron spectra from collisions of $80 \mathrm{MeV} \mathrm{O}^{8+}$ with $\mathrm{Ne}$ at $\theta_{0}=0.4^{\circ}(a)$ and $1.2^{\circ}(b)$. Experiment, dots. $B_{n}$ fit to the spectra, full curve. Constructed spectrum for $0.4^{\circ}$ with the $B_{n,}$ from $1.2^{\circ}$, broken curve. Post IA normalized to the cusp maximum, chain curve.

into the expression (6.5) for $\theta_{0}=1.2^{\circ}$, the so constructed spectrum severely disagrees with the measured one at $\theta_{0}=1.2^{\circ}$ (figure $5(b)$ ).

The situation is similar for $80 \mathrm{MeV}$ impact. In figure 6 we show the two spectra for $\theta_{0}=0.4^{\circ}$ and $1.2^{\circ}$ and their respective fits, as well as the results from the post IA at $1.2^{\circ}$. For the two shape parameters characterizing the vicinity of the cusp maximum, $\beta_{1}=B_{01} / B_{00}$ and $\beta_{2}=B_{02} / B_{00}$, we have obtained $\beta_{1}=-0.329, \beta_{2}=-0.268$ for $0.4^{\circ}$ and $\beta_{1}=-0.427, \beta_{2}=-0.017$ for $1.2^{\circ}$ while the post IA predicts a somewhat larger asymmetry, $\beta_{1}=-0.665$ and $\beta_{2}=-0.08$. If the $B_{n i}\left(0.4^{\circ}\right)$ are used to construct a spectrum at $1.2^{\circ}$ by means of $(6.5)$, the disagreement with the measured spectrum at $1.2^{\circ}$ is even worse than in case of $40 \mathrm{MeV}$ impact. If, on the other hand, a spectrum at $0.4^{\circ}$ is constructed from the $B_{n i}\left(1.2^{\circ}\right)$, the disagreement with the experimental data is less severe, but still significant (figure $6(a)$ ).

Due to these features of the $B_{n t}$ parameters we have preferred to use the width for the characterization of the forward peak shape. As has been demonstrated by Day (1980), the full width at half maximum, $\Gamma_{\mathrm{FWHM}}$, scales with $\theta_{0}$ according to

$$
\Gamma_{\mathrm{FWHM}}=\frac{3}{2} \theta_{0} v\left(1+\frac{3}{2} \beta_{2}\right)
$$




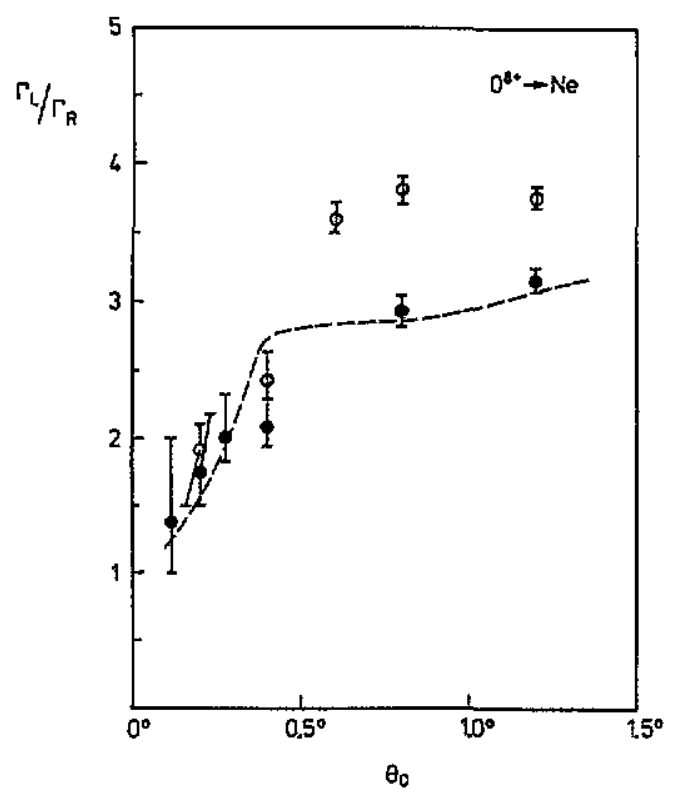

Figure 7. Ratio between left $\left(\Gamma_{L}\right)$ and right $\left(\Gamma_{R}\right)$ half-width at half-maximum of the forward peak for $\mathrm{O}^{8+}$ on $\mathrm{Ne}$ as a function of detector resolution $\theta_{0}$. Full symbols, data for $40 \mathrm{MeV}$ impact; open symbols, data for $80 \mathrm{MeV}$ impact; broken curve, post iA for $80 \mathrm{MeV}$ impact.

where the theoretical value for $\beta_{2}$ is independent of $\theta_{0}$ but may depend on $v$. This scaling of $\Gamma_{\text {FWHM }}$ with $\theta_{0}$ at fixed $v$ is well satisfied in our experiments (Oswald 1989).

The asymmetry of the cusp may be characterized by the ratio of the half widths at half maximum on the low-energy side $\left(\Gamma_{L}\right)$ and on the high-energy side $\left(\Gamma_{R}\right)$ of the peak. The ratios $\Gamma_{\mathrm{L}} / \Gamma_{\mathrm{R}}$ for $40 \mathrm{MeV}$ and $80 \mathrm{MeV} \mathrm{O}^{8+}$ colliding with $\mathrm{Ne}$ are shown in figure 7 as a function of $\theta_{0}$. At small $\theta_{0}$ there is a steep rise of $\Gamma_{\mathrm{L}} / \Gamma_{\mathrm{R}}$ with $\theta_{0}$ which flattens out for $\theta_{0} \geq 1^{\circ}$.

From a $\theta_{0}$-independent shape parameter $\beta_{1}$ one expects a half-width ratio which is roughly independent of $\theta_{0}$ because the $\theta_{0}$ dependence of (6.6) should drop out. (If only $B_{00}$ and $\beta_{1}$ are included in the series (6.2) an average over $\theta_{0}$ provides a balf-width ratio $\Gamma_{L} / \Gamma_{R}$ which shows a weak linear decrease with $\theta_{0}$-below $5 \%$ for $0.25^{\circ} \leqslant 0_{0} \leqslant 1.2^{\circ}$ in the $\beta_{1}$ region investigated, $-0.5 \leqslant \beta_{1} \leqslant-0.25$ ). Our measured $\Gamma_{L} / \Gamma_{R}$ are, however, only constant-within the experimental uncertainty-for $\theta_{0} \geqslant 1^{\circ}$. The strong decrease of the half-width ratio for $\theta_{0} \rightarrow 0$ in our experiments is therefore another indication of a $\theta_{0}$ dependence of the shape parameters $B_{n j}$. Theoretical calculations within the post IA confirm the experimental results (figure 7). Due to numerical convergence problems near the cusp maximum, the calculated ratios may have uncertainties from $10 \%$ at $\theta_{0} \geqslant 1^{\circ}$ to $30 \%$ at $\theta_{0} \leqslant 0.4^{\circ}$. Also previous second-order Born calculations (without accounting for target screening) confirm the decrease of $\Gamma_{\mathrm{L}} / \Gamma_{\mathrm{R}}$ for $\theta_{0} \rightarrow 0$ (Oswald et al 1989).

The velocity dependence of the half-width ratio is plotted in figure 8 . For hydrogen impact the peak asymmetry is very small $\left(\Gamma_{L} / \Gamma_{R} \sim 1.2\right)$ and approximately constant in the velocity region considered. The experimental results are well described by the second Born theory as well as by the prior impulse approximation, the $\mathrm{B} 2$ being somewhat superior. The asymmetry in the (fully peaked) ${ }_{1} \mathrm{~A}$ is solely due to the smooth $k_{\mathrm{f}}$ dependence of the 'background' part of the cross section (i.e. of $d^{2} \sigma^{\mathrm{I}} / \mathrm{d} E_{\mathrm{f}} \mathrm{d} \Omega_{\mathrm{f}}$ in (4.2)). For 


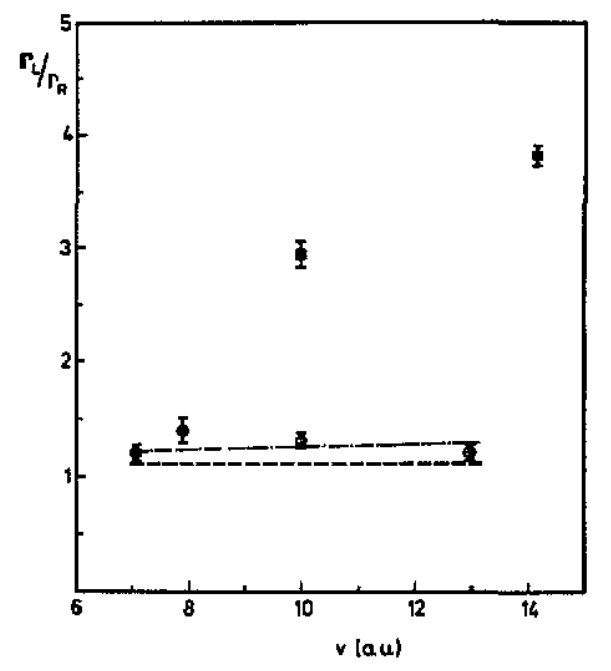

Figure 8. Ratio between left $\left(\Gamma_{\mathrm{L}}\right)$ and right $\left(\Gamma_{R}\right)$ half-width at half-maximum of the forward peak for $\mathrm{H}^{+}$and $\mathrm{O}^{8+}$ on Ne as a function of collision velocity. Experiment: $\varnothing_{1}, \mathrm{H}^{+}$projectiles with $\theta_{0}=0.75^{\circ} ; \mathbf{9}, \mathrm{O}^{8+}$ projectiles with $\theta_{0}=0.75^{\circ} ; \mathrm{O}^{8+}$ projectiles with $\theta_{0}=0.8^{\circ}$. Theory: chain curve, B2 for $\mathrm{H}^{+}$and $\theta_{0}=0.75^{\circ}$; broken curve, prior $\mathrm{IA}$ for $\mathrm{H}^{+}$and $\theta_{0}=0.75^{\circ}$.

$\mathrm{O}^{8+}$ projectiles, on the other hand, an increase of the collision velocity from $v=8$ to $v=14$ leads to a strong increase of the peak asymmetry for fixed $\theta_{\mathrm{a}}$. This shows that even at the highest velocity one is still far from the asymptotic regime where the perturbative theoretical approaches (like B2 or IA) are valid, i.e. where the asymmetry decreases with velocity. (The iA gives a slightly larger ratio $\Gamma_{\mathrm{L}} / \Gamma_{\mathrm{R}}$ at the lower energy, $40 \mathrm{MeV}$, as compared to $80 \mathrm{MeV}$.) The fact that for high (but fixed) velocity the asymmetry for oxygen impact is much larger than for hydrogen impact can nevertheless be interpreted within the high-energy theories: from the representation (6.4) of the final-state Coulomb wave it is readily seen that the phase which causes the asymmetry, increases with the projectile charge $Z_{\mathrm{P}}$.

\section{Final remarks and conclusion}

We have measured zero-degree electron spectra resulting from ECC in the collision systems $\mathrm{H}^{+}$and $\mathrm{O}^{8+}$ on $\mathrm{Ne}$ in the velocity region $0.7 \leqslant v / Z_{\mathrm{T}} \leqslant 1.4$. For proton impact, all cusp spectra are only weakly asymmetric. Since the collision parameters for this system are mostly in the validity regime of the prior impulse approximation $\left(Z_{\mathrm{P}} \ll Z_{\mathrm{T}}\right.$, $\left.v / Z_{\tau}>1\right)$, this theory gives a satisfactory description of both peak intensity and shape, except at the lowest velocity. As far as the second-order Born approximation is concerned, we have extended its validity regime from $Z_{\mathrm{P}} \sim Z_{\mathrm{T}}, v / Z_{\mathrm{T}} \gg 1$ to lower velocities by omitting additional peaking approximations. Nevertheless, the strong asymmetry of the $\mathrm{H}^{+}+\mathrm{Ne}$ collision system leads still to an overprediction of the experimental peak intensities for $v / Z_{\mathrm{T}} \gtrsim 1$, whereas the peak shapes are well described within $\mathrm{B} 2$.

For oxygen impact we have found a considerable skewness of the forward peak towards the low-energy side which increases with collision velocity. For this system the impact velocities are too low for perturbative theories such as the IA or the B2 to be valid; indeed, both theories drastically overestimate the measured cusp intensity at all 
velocities. Nevertheless, the post IA as well as the B2 (Oswald 1989) give a reasonable account of the peak shape at higher velocities.

One of our main goals was the investigation of the peak shape when the detector angular resolution is improved beyond the values commonly used. Since for proton impact the peak asymmetry and hence the peak shape dependence on $\theta_{0}$ is small in our velocity regime, we have restricted ourselves to study the peak asymmetry for oxygen impact when $\theta_{0}$ is varied. We have found a strong dependence of the shape parameters $B_{n l}$ on $\theta_{0}$. This feature is confirmed by an equally strong $\theta_{0}$ dependence of the cusp asymmetry as measured by the half-width ratio $\Gamma_{\mathrm{L}} / \Gamma_{\mathrm{R}}$ for $\theta_{0}<1$. This $\theta_{0}$ dependence of $\Gamma_{L} / \Gamma_{R}$ is supported by both the post LA and the second-order Born theory and can be traced back to a non-analytic behaviour of the differential cross section in terms of the projectile-frame emission angle at the cusp maximum.

After completion of our experiments, Berényi et al (1991) and Gulyás et al (1991) have also studied the $\theta_{0}$ dependence of the shape parameters for $0.2-0.3 \mathrm{MeV} \mathrm{H}^{+}$impact on $\mathrm{He}\left(v / Z_{\mathrm{T}} \sim 1.5\right)$ and $0.3^{\circ} \leqslant \theta_{0} \leqslant 2^{\circ}$. Similar to our results, they have found a decrease of the cusp asymmetry with decreasing $\theta_{0}$, expressed in a decrease of $\left|\beta_{1}\right|$ as well as $\Gamma_{\mathrm{L}} / \Gamma_{\mathrm{R}}$. Only the strong decrease of $\Gamma_{\mathrm{L}} / \Gamma_{\mathrm{R}}$ at $\theta_{0} \leqslant 0.6^{\circ}$, which we found for $\mathrm{O}^{8+}$ on Ne, is absent in their $\mathrm{H}^{+}$on He data. Believing in the $\theta_{0}$ independence of the $B_{n l}$ parameters, they argued, however, that there are ambiguities in the determination of $\theta_{0}$ from the geometry of the set-up. When including $\theta_{0}$ as a fit parameter together with the $B_{n t}$ they found a much weaker increase of $\left|\beta_{1}\right|$ with $\theta_{0}$ which even-within the experimental errors-could be reconciled with the assumption of a $\theta_{0}$-independent $\beta_{1}$. In this context we should like to note that our method for the determination of $\theta_{0}$ is much more precise than the pure geometrical considerations of Gulyás et al (1991). In conjunction with the theoretical argumentation from above we therefore conclude that a fit of the ECC spectra to a truncated $B_{n l}$ expansion does not lead to generic $B_{n l}$ values which are independent of the experimental set-up, particularly for very small $\theta_{0}$.

\section{Acknowledgments}

We should like to thank L Gulyás and Gy Szabó for helpful discussions and the GSI Darmstadt for partial support of this work.

\section{Appendix}

We evaluate the Dalitz-type integral (Dalitz 1951) from (3.11) for arbitrary parameters with $\alpha, \beta \geqslant 0$ and $q_{i}, q_{\mathrm{f}}, k^{2}$ real. Let

$$
I_{0}=\int \mathrm{d} k^{\prime} \frac{1}{k^{\prime 2}-k^{2}-\mathrm{i} \varepsilon} \frac{1}{\alpha^{2}+\left(k^{\prime}-q_{\mathrm{i}}\right)^{2}} \frac{1}{\beta^{2}+\left(k^{\prime}-q_{\mathrm{f}}\right)^{2}}
$$

with $\varepsilon \rightarrow 0$. With the help of the Feynman identity for the denominators in (A.1) involving $\alpha^{2}$ and $\beta^{2}$

$$
\frac{\mathrm{l}}{a b}=\int_{0}^{1} \frac{\mathrm{d} t}{[a t+b(1-t)]^{2}}
$$


one obtains

$$
\begin{aligned}
& I_{0}=\int_{0}^{1} \mathrm{~d} t \int \mathrm{d} k^{\prime} \frac{1}{\left(k^{\prime 2}-k^{2}-\mathrm{i} \varepsilon\right)\left[\Gamma^{2}+\left(k^{\prime}-\Lambda\right)^{2}\right]^{2}} \\
& \Gamma^{2}(t)=\alpha^{2} t+\beta^{2}(1-t)+\left(q_{\mathrm{f}}-q_{\mathrm{i}}\right)^{2}\left(t-t^{2}\right) \\
& \Lambda(t)=q_{\mathrm{f}}-t\left(q_{\mathrm{f}}-q_{\mathrm{i}}\right) .
\end{aligned}
$$

The integral over $\boldsymbol{k}^{\prime}$ can be evaluated by means of contour integration (Joachain 1983) such that $I_{0}$ reduces to

$$
I_{0}=-\pi^{2} \int_{0}^{1} \frac{\mathrm{d} t}{\left(k^{2}-\Gamma^{2}-\Lambda^{2}+2 \mathrm{i} k \Gamma\right) \Gamma} .
$$

Inserting $\Gamma^{2}(t)$ and $\Lambda^{2}(t)$ into the denominator of (A.4) and using the definitions (3.11) one obtains

$$
\begin{aligned}
& I_{0}=-\pi^{2}(F(1)-F(0)) \\
& F(t)=\int^{t} \frac{\mathrm{d} t}{\left[\left(\gamma_{\mathrm{f}}-\gamma_{\mathrm{i}}\right) t-\gamma_{\mathrm{f}}+2 \mathrm{i} k \Gamma\right] \Gamma} .
\end{aligned}
$$

The integral over $t$ can be done by elementary integration techniques after multiplication of denominator and numerator by the complex conjugate of the expression in square brackets in (A.5). One obtains the result

$$
F(t)=\frac{2 \mathrm{i} k}{c\left(p_{+}-p_{-}\right)} \ln \frac{\mu_{-} t+\delta_{-}+\Gamma(t)}{\mu_{+} t+\delta_{+}+\Gamma(t)}
$$

In this expression, $p_{+}$and $p_{-}$are the roots of the quadratic equation

$$
\left[\left(\gamma_{\mathrm{f}}-\gamma_{\mathrm{i}}\right) t-\gamma_{\mathrm{f}}\right]^{2}+4 k^{2} \Gamma^{2}(t)=0
$$

while the other quantities which involve the coefficients of the powers in $t$ of $\Gamma^{2}(t)$, are defined by

$$
\begin{aligned}
& \mu_{ \pm}=\frac{1}{2 b_{ \pm}}\left[\alpha^{2}-\beta^{2}+\left(q_{\mathrm{f}}-q_{\mathrm{i}}\right)^{2}-\left(q_{\mathrm{f}}-q_{\mathrm{i}}\right)^{2} p_{ \pm}\right] \\
& \delta_{ \pm}=\frac{1}{2 b_{ \pm}}\left[2 \beta^{2}+\left(\alpha^{2}-\beta^{2}+\left(q_{\mathrm{f}}-q_{\mathrm{I}}\right)^{2}\right) p_{ \pm}\right] \\
& b_{ \pm}=\frac{1}{2 \mathrm{i} k}\left[\left(\gamma_{\mathrm{f}}-\gamma_{\mathrm{i}}\right) p_{ \pm}-\gamma_{\mathrm{f}}\right] .
\end{aligned}
$$

Insertion of (A.6) into (A.5) results after a lengthy calculation in the simple result

$$
I_{0}=\frac{\mathrm{i} \pi^{2}}{2 \sqrt{\Delta}} \ln \left(\frac{a-\mathrm{i} \sqrt{\Delta}}{a+\mathrm{i} \sqrt{\Delta}}\right)^{2}
$$

where $a$ and $\Delta$ are defined in (3.11).

\section{References}


Breinig M, Elston S B, Huldt S, Liljeby L, Vane C R, Berry S D, Glass G A, Schauer M, Sellin I A, Alton G D, Datz S, Overbury S, Laubert R and Suter M 1982 Phys. Rev. A 253015

Briggs J S 1977 J. Phys. B: At. Mol. Phys. 103075

Burgdörfer J 1986 Phys, Rev. A 331578

Cranage R W and Lucas M W 1976 J. Phys. B: At. Mol. Phys. 9445

Crothers D S F and McCann J F 1987 J. Phys. B: At. Mol. Opt. Phys, 20 L19

Dalitz R H 1951 Proc. R. Soc. A 206509

Day M H 1980 J. Phys. B. At. Mol. Phys. 13 L65

Dettmann K, Harrison K G and Lucas M W 1974 J. Phys. B: At. Mol. Phys. 7269

Enge H 1967 Focussing of Charged Particles ed A Septier (New York: Academic)

Garibotti C R and Miraglia J E 1980 Phys. Rev. A 21572

Groeneveld K-O, Meckbach W and Sellin I A (ed) 1984 Forward Electron Ejection in Fon-Atom Collisions (Lecture Notes in Physics 213) (Berlin: Springer)

Gulyás L, Kövér Á, Szabó Gy, Vajnai T and Berényi D 1991 Lecture Notes in Physics 376 (Berlin: Springer) p 56

Gulyás L, Szabó Gy, Berényi D, Kövér A, Groeneveld K-O, Hofmann D and Burkhard M 1986 Phys. Rev. A 342751

Jakubassa-Amundsen D H 1983 J. Phys. B: At. Mol. Phys. 161767

1988 Phys. Rev. A 3870

Joachain C J 1983 Quantum Collision Theory (Amsterdam: North Holland) appendix D

Knudsen H, Andersen L J and Jensen K E 1986 J. Phys. B: At. Mol. Phys. 193341

Lucas M W and Steckelmacher W 1987 Lecture Notes in Physics 294 (Berlin: Springer) p 229

Macek J 1970 Phys. Rev. A 1235

Macek J, Potter J E, Duncan M M, Menendez M G, Lucas M W and Steckeimacher W I981 Phys. Rev. Lett. 461571

Meckbach W, Nemirovsky I B and Garibotti C 1981 Phys. Rev. A 241793

Menendez M G, Duncan M M, Eisele F L and Junker B R 1977 Phys. Rev. A 1580

Miraglia J E and Ponce V H 1980 J. Phys. B: At. Mol. Phys. 131195

Moiseiwitsch B L $1991 \mathrm{~J}$. Phys. B; At. Mol. Opt. Phys. 24983

Newton R G 1966 Scattering Theory of Waves and Particles (Berlin: Springer) ch 16.3 .2

Oswald W 1989 Thesis University of Munich

Oswald W, Schramm R and Betz H-D 1989 Phys. Rev. Lert. 621114

Rødbro M and Andersen F D 1979 J. Phys. B; At. Mol. Phys, 122883

Rudd M E, Sautter C A and Bailey C L 1966 Phys. Rev. 15120

Salin A 1969 J. Phy's. B: As. Mol. Phys. 2631

Shakeshaft R and Spruch L 1978 Phys. Rev, Lett. 411037

Stolterfoht N 1978 Topics in Current Physics vol 5 ed I A Sellin (Berlin: Springer) pp 155-99 